\title{
INVESTIGATION OF INLET DISTORTION EFFECTS ON AXIAL COMPRESSOR PERFORMANCE BASED ON STREAMLINE CURVATURE METHOD
}

\author{
Sarallah AbBasi \\ Department of Mechanical Engineering, Arak University of Technology, Arak, Iran \\ e-mail:s_abbasi@arakut.ac.ir \\ AlirezA PIRNiA \\ Department of Mechanical Engineering, Clarkson University, New York, USA \\ Reza Taghavi-Zenouz \\ Department of Mechanical Engineering, Iran University of Science and Technology, Tehran, Iran
}

\begin{abstract}
In this paper, the effects of inlet flow distortion on performance maps and details of the flow field are investigated using the Streamline Curvature Method. The results are presented for both design and off-design conditions and compared with experimental data, which shows good agreement. The effects of inlet flow distortion are investigated by inlet total pressure variation in three different cases in the way that the average total pressure remains constant and equal to the design value. The results show that a relative increase in the total pressure at tip causes an increase in the pressure ratio and efficiency as well as a better performance in the choking region. Alternatively, a relative increase in the total pressure in hub causes opposite behavior and a better performance at the surging region.
\end{abstract}

Keywords: axial compressor, performance map, inlet distortion, surge margin, streamline curvature

\section{Introduction}

The compressor is one of the most important parts of jet engines which must have a stable operation in all flight maneuvers or atmospheric conditions. One of the most appropriate assumptions in the normal approach to compressor and fan design is undistorted flow at the inlet in which flow properties are assumed to be uniform and the flow direction to be axial. But under a variety of conditions in military and commercial aircraft engines, a highly distorted flow at the compressor inlet is produced, which is commonly referred to as "inlet distortion". Non uniform flow at the inlet of the fan stage may be originated from atmospheric turbulence, boundary layer growth, flow distortions induced by the shape of the intake duct and boundary layer ingesting engines in the modern high efficient airframe.

Many performance problems and engine failures in aircraft including rotating stall and surge were observed due to inlet distortion. Therefore, it is important to explore the behavior of the engine compressor towards inlet distortion. There are many ways to investigate the flow field in an axial compressor or fan with inlet distortion. In prior research investigations, models of the response to distortion have been developed which utilize clean compressor performance or stage characteristics to anticipate the compressor behavior with distortion (Chue et al., 1989; Day, 1993; Greitzer, 1980).

With rapid development of computer technology, Computational Fluid Dynamics (CFD) methods have been employed extensively to study the flow field in a compressor with inlet flow distortion. Zhang and Hou (2017) explored stall inception in a low-speed axial compressor under 
rotating distorted inflow conditions using full annulus, unsteady, three dimensional computational fluid dynamics (CFD). The results indicated that under co-rotating inflow distortion, close to the tip region the flow incidence angle is increased and the axial flow coefficient decreased. It has an important role on the stall inception process. Liu et al. (2016) investigated the effect of inlet distortion on the performance of an axial transonic contra-rotating compressor by means of full-annulus simulation. Results revealed that the inlet distortion leads to reduction of the pressure ratio and efficiency. The interaction between the detached shockwave and the tip leakage flow leads to blockage of the blades passage. It is one important of reasons for the compressor stall. Naseri and Boroomand (2016) using CFD approach evaluated the effect of inlet distortion in forms of inlet swirl and total pressure distortion on compressor performance and stability. Results indicated that hub and tip radial distortions change the stability range of the compressor.

Review of the literature shows that most of the researches were employed experimentally and by numerical methods (CFD method) to explore the effects of inlet distortion on axial compressor performance. In experimental tests, limitations of instruments and cost make this method difficult to investigate. Also, application of CFD analysis, which is associated with solving complete flow and energy equations in a very complex geometry like a compressor or fan, takes long period of time and is not reasonable.

Due to difficulties in experimental and CFD analysis, various methods with some simplifications are presented for design purposes and flow analysis. Some researchers such as Kim et al. (1996) used a simple integral method which rendered the multistage analysis as a natural part instead of solving a detailed flow field problem. By using this method, they calculated the qualitative trend of distorted performance of an axial compressor successfully. Most of researchers and designers prefer working on meridional plane of a turbomachine (Lakshminarayana, 1996). Governing equations on hub to tip through the flow surface (referred to as S2-surface) can be solved on the meridional plane using streamline curvature (SCM). This method is mostly used because of its rapid convergence and accurate results. Final results based on this method are comparable with those obtained through CFD methods, which consume usually extreme time and expenses (Pachidis and Pilidis, 2006). This method has been employed by Hearsey (1986), Pachidis and Pilidis (2006), Hu et al. (2011) and Gong et al. (2016) to design and analyze different kinds of turbomachines.

Based on the above mentioned subjects, it can be realized that the effects of inlet distortion on the compressor performance are investigated, but details of characteristics of flow fields such as flow angles, velocity and pressure field are still under question. In this paper, the streamline curvature method, as a rapid and accurate method, is used to investigate the effects of inlet total pressure distortion on flow field details, performance characteristics and surge/choke margins.

\section{Streamline Curvature Method and verification}

\subsection{Streamline Curvature Method}

Governing equations for viscous compressible flows through compressors are highly nonlinear. Therefore, most of methods that have been presented so far utilize some simplifications. One effective approach is to divide the flow field into a series of blade to blade surfaces (so-called S1-surface) and some surfaces extended from hub to tip (so-called S2-surface). Based on the experiences, working only on the through-flow surface, i.e., the S2-surface, which is extended from the hub to tip in the radial and mean flow directions, can provide the final geometry of blades with an acceptable accuracy. The Streamline Curvature Method (SCM) is widely used for quasi three-dimensional design of axial compressor blades. This method which was presented 
by Novak (1967) is based on successive refinement of streamlines to satisfy the balance between centrifugal and pressure forces while ensuring the given mass flow rate.

SCM utilizes primarily an inviscid flow. In fact, it offers a flexible method of determining the Euler solution of axisymmetric flow through a turbomachine. Then, a discrete increase of entropy, which is determined from loss correlations, is imposed to represent viscous effects. It is obvious that accuracy of results highly depends on the validity of loss correlations. The governing main system of equations consists of linear momentum and continuity equations. Then, combined with the thermodynamic equation of state, the resultant equation is mapped into the meridional plane of the flow passage. As a consequence of the assumptions made in this method, and mapping into the meridional plane, the final form of the governing equation can be presented as Eq. (2.1). Some of the parameters appeared in this equation are introduced in Fig. 1, showing the coordinate axes and angles necessary for formulation in SCM. Fundamentals of this method including its precise concept and more details on the governing equations and necessary correlations can be found in Hearsey (1986)

$$
V_{m} \frac{d V_{m}}{d l}=\sin (\varphi-\gamma)\left(V_{m} \frac{\partial V_{m}}{\partial m}-F_{m}\right)+\cos (\varphi-\gamma)\left(\frac{V_{m}^{2}}{r_{c}}-F_{n}\right)+\frac{d h_{0}}{d l}-T \frac{d s}{d l}-\frac{V_{\theta}}{r} \frac{d\left(r V_{\theta}\right)}{d l}
$$

where $V_{m}$ and $V_{\theta}$ are, respectively, the meridional and swirl velocity, $F_{m}$ and $F_{n}$ are blade forces (meridional blade force and normal blade force), $l$ and $m$ are, respectively, the quasi-orthogonal and meridional direction, $r$ and $r_{c}$ is the radial direction and streamline curvature radius, $h_{0}$ is stagnation enthalpy, $s$ is entropy and $T$ is static temperature. $\varphi$ and $\gamma$ are deflection and sweep angles.

Equation (2.1) is solved as an ordinary first order differential equation. In this respect, a computerized program is developed which consists of two main parts. The first part is mainly attributed to obtain three dimensional geometry of blades, and the next part produces performance curves of the turbomachine. The computer code utilizes empirical correlations for calculations of various kinds of loss accompanied by axial compressors. Obviously, reliability of final results depends highly on the degree of precision of these correlations.

\subsection{Loss and angle correlations}

In order to implement viscous effects in governing equations, empirical correlations must be used for the loss coefficient, incidence and deviation angles. The loss coefficient is divided into four parts; profile loss, shock loss, secondary flow loss and tip clearance loss. The profile loss coefficient is calculated from Koch and Smith's correlation (1976), shock loss coefficient from Swan's correlation (1958), secondary flow loss coefficient from Griepentrog's correlation (1970) and tip clearance loss coefficient from Storer and Cumpsty's correlation (1994). These coefficients are based on the minimum loss condition (design condition). Therefore, summation of these four coefficients is multiplied by a factor which includes off-design effects in the total loss coefficient. This factor is based on the difference between design and off-design incidence angles. Equation (2.2) shows the total loss coefficient for each blade row

$$
\omega=\left(\omega_{p}+\omega_{s h}+\omega_{s e c}+\omega_{t i p, c l}\right) \frac{i-i_{\text {des }}}{W^{2}}
$$

where $\omega_{p}$ is the blade profile loss factor, $\omega_{s h}$ is the blade shock wave loss factor, $\omega_{\text {sec }}$ is the secondary loss factor, and $\omega_{t i p, c l}$ is the tip clearance loss factor. In this equation, $i_{\text {des }}$ and $i$ are the design and off design incidences, respectively. An increment in the loss of actual incidence (off-design) and Mach number is originally accounted for through the parabolic term in brackets in Eq. (2.2). $W$ in Eq. (2.2) is "width" which specifies the working range of the section. This is a common approach taken in streamline curvature methods to achieve a more realistic loss 
"bucket" shape (Cetin et al., 1987). Other parameters which are computed via empirical correlations are incidence and deviation angles. Lieblein's popular correlations are used for obtaining these parameters (Cumpsty, 1989).

\subsection{Surge and choking criteria}

In order to obtain the mass flow rate in which surge or choking occurs in each speed line, some criteria must be defined. One suitable criterion for surge or stall occurrence, which is used in the current research, belongs to Aungier (2003). In his method, the aerodynamic velocity ratio across each blade row $\left(W_{\text {aero }}\right)$ and the equivalent geometric velocity ratio $\left(W_{\text {geom }}\right)$, which are calculated from equation (2.3), are compared (Aungier, 2003)

$$
W_{\text {aero }}=\sqrt{\frac{P_{0,2}-P_{2}}{P_{0,1}-P_{1}}} \quad W_{\text {geom }}=\frac{\frac{0.15+11 \frac{t_{\max }}{c}}{0.25+10 \frac{t_{\max }}{c}}}{1+0.4\left(\frac{\theta \sigma}{2 \sin \frac{\theta}{2} \cos \gamma}\right)^{0.65}}
$$

where $t_{\max }$ represents the maximum thickness, $c$ is the chord length, $\gamma$ is the stagger angle, $\sigma$ is the solidity, $\theta$ is the momentum thickness, $P_{0,2}$ and $P_{0,1}$ are the total pressures downstream and upstream the blade row, respectively. Also, $P_{2}$ and $P_{1}$ are the static pressures downstream and upstream the blade row, respectively. $W_{\text {geom }}$ is just a function of geometric parameters and, therefore, remains constant for varying mass flow rate while $W_{\text {aero }}$ increases as the flow rate decreases. Aungier (2003) suggested that as long as the aerodynamic velocity ratio remains higher than the geometric velocity ratio, the compressor is un-stalled, or surge has not occurred. In order to predict choking in the compressor, a decrease in the rate of Mach number variation is considered as the beginning of choking.

\subsection{Experimental validation}

In the design of an axial fan or compressor by the streamline curvature method, several input parameters such as meridional geometry, inlet condition, radial distribution of some parameters, and etc. are required. Also, to validate SCM results, experimental results in forms of radial distribution of parameters, performance maps and flow fields (meridional velocity and etc.) are required. To validate results in design and off-design conditions, Urasek et al. (1979) employed experimental data. Although that paper (Urasek's fan) is a very old one, but it is one of the best papers that provides all required data for design of an axial fan by SCM. These data refer to a two-stage axial fan with a pressure ratio of 2.4. A view of its blades in the meridional plane along with the computational grid is shown in Fig. 1.

Overall characteristics of this fan are also shown in Table 1. Also, the estimated errors of the experimental data based on inherent accuracies of the instrumentation and recording system are provided in the paper (Urasek et al., 1979) and added to Table 1.

Table 1. Overall characteristics of Urasek's two-stage fan (Urasek et al., 1979)

\begin{tabular}{|l|c|}
\hline Inlet total pressure $[\mathrm{kPa}]$ & $101.325 \pm 0.7$ \\
\hline Inlet total temperature $[\mathrm{K}]$ & $288 \pm 0.6$ \\
\hline Mass flow rate $[\mathrm{kg} / \mathrm{s}]$ & $33.2 \pm 0.3$ \\
\hline Rotational velocity $[\mathrm{r} / \mathrm{min}]$ & $16042 \pm 30$ \\
\hline Overall pressure ratio & 2.4 \\
\hline Number of stages & 2 \\
\hline First rotor aspect ratio & 1.56 \\
\hline Aerodynamic efficiency $[\%]$ & 84.9 \\
\hline
\end{tabular}




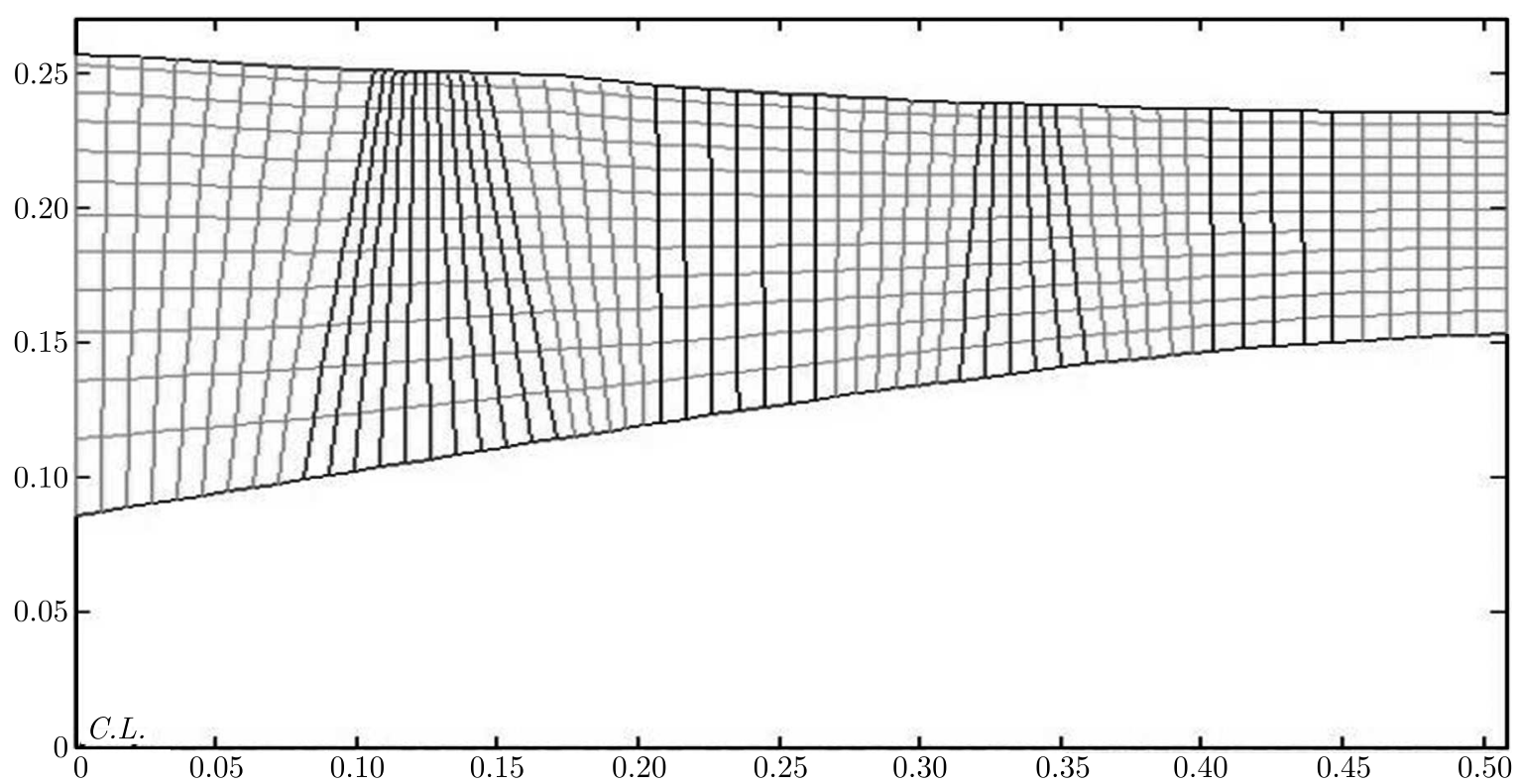

Fig. 1. Computational grid in the meridional plane

Analyzing the results of design conditions is not in the scope of this paper. But in Fig. 2, as an example, the meridional velocity distribution (as the most important result of SCM) for the first rotor is compared with the experimental one. In this figure, the difference between the obtained results with the experimental ones is about $8 \%$.

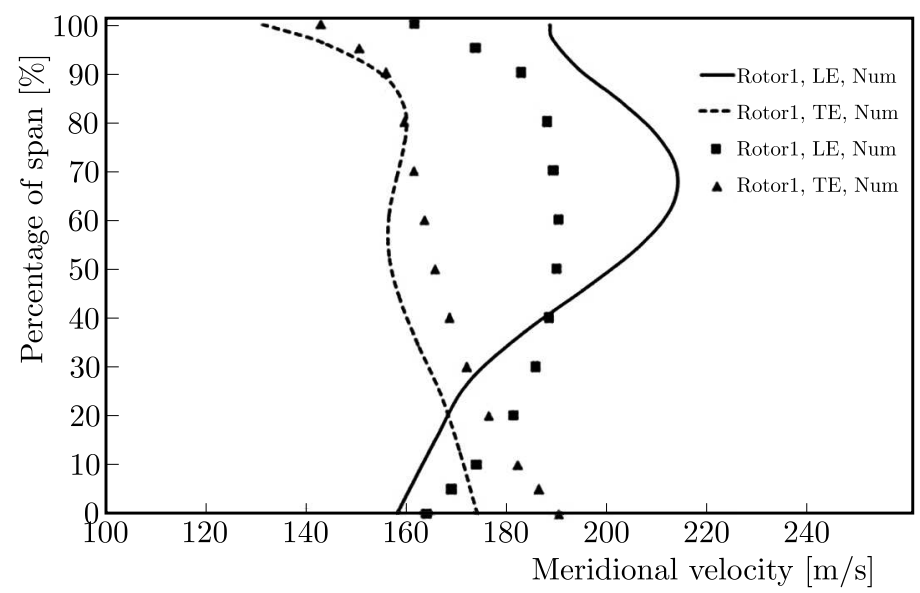

Fig. 2. Experimental validation of the first rotor meridional velocity in the design condition

The performance characteristics taken from the presented research work against the experimental ones are shown in Figs. 3 and 4. Figure 3 shows the variation of pressure ratio in 100 to 50 percent speed lines. The chance of choking occurrence is increased with increasing rotational speed. As shown in Fig. 3a, the maximum discrepancy between the SCM result and experimental data is lower than $7 \%$ in each speed line. Referring to Fig. 3b, good agreement is observed between SCM results and experimental data, and the maximum discrepancy is about $5 \%$ in $100 \%$ speed line. 
(a)

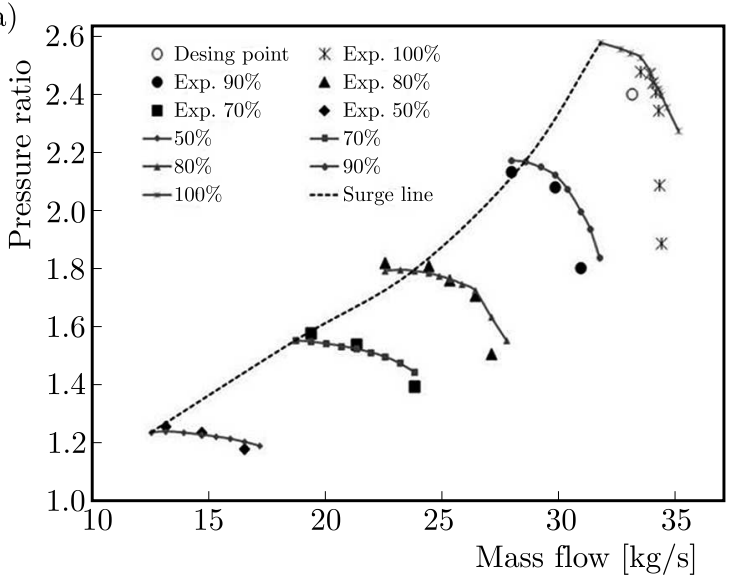

(b)

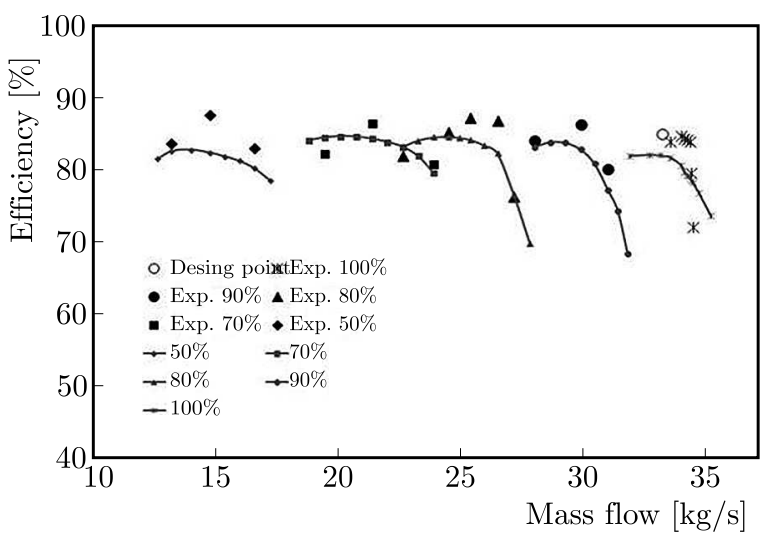

Fig. 3. Experimental validation of (a) fan pressure ratio and (b) fan efficiency in the off-design condition

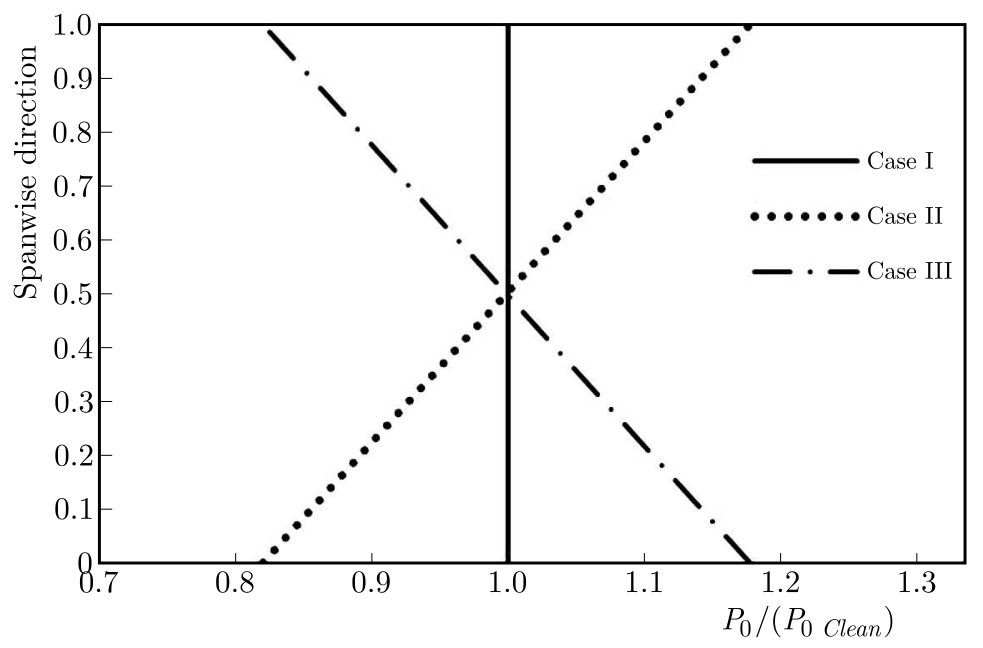

Fig. 4. All cases of disturbance patterns

\section{Results and discussion}

During flights, the inlet total pressure distortion due to rapid maneuvering or bumping into strong cross-winds causes destabilization of aircraft engines. The aerodynamic stability problems of compressors become increasingly critical while the loads of modern compressors are ceaselessly increasing. Conventional, good prediction of the performance of a whole engine can be achieved by a low-fidelity gas turbine performance simulation tool. But it is incapable of both precisely exploring the performance of individual engine components and grasping complex physical phenomena such as inlet flow distortion. On the other hand, CFD tools can study the performance of individual engine components appropriately, but application of CFD analysis, which is associated with solving complete flow and energy equations in very complex geometry typical for compressors or fans, takes a long period of time and is not reasonable. Also, experimental tests, due to limitations of measurement instruments and cost make this method difficult for investigation. Therefore, in the present work, the streamline curvature method (SCM) has been used to model inlet flow distortion due to fast convergence, precision results and the absence of the above constrains. As shown above, the presented results are comparable with those obtained from experimental tests.

Occurrence of distortion patterns in the incoming flow is applied in simulations by defining proper boundary conditions at the inlet. In the current research, the inlet flow with total pressure distortion is studied. Radial variations are in a way that the average total pressure 
remains constant and equal to design value. Accordingly, a parameter named "distortion index" is introduced as follows (Aungier, 2003)

$$
I=\frac{P_{0, \max }-P_{0, \min }}{P_{0, \max }}
$$

where $P_{0, \max }$ and $P_{0, \min }$ are the maximum and minimum total pressures. All considered cases are shown in Fig. 4. Case I has the design condition with a uniform inlet total pressure. Case II has linear variation of the inlet total pressure with the maximum at the tip and the minimum in the hub. Case III is opposite to the second case. The distortion index in the second and third cases is equal to 0.3. But in this regard, other researchers behaved differently. For example, Naseri and Boroomand (2016) used a CFD approach to investigate the effect of radial total pressure disturbances but the average total pressure was not the same in different cases. In another research, Liu et al. (2016) using commercial CFD software investigated the effects of circumferential total pressure inlet distortion on the performance and flow field of the axial counter-rotating compressor but the radial disturbance was not studied.

As can be seen in Fig. 5, the total pressure increase at the tip (case II) aims at blade loading and is more successful in controlling the loss in this region than in other cases. This fact leads to an increase in the pressure ratio and efficiency. Also, in this case, a lower total pressure in the hub make the Mach numbers lower at this region, which produces choking at higher mass flow rates.

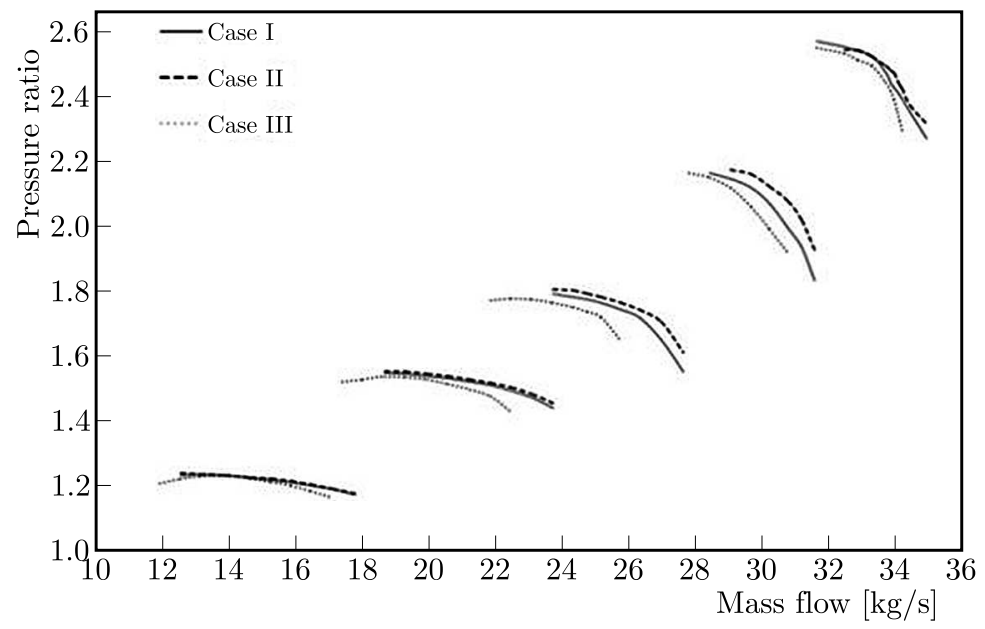

Fig. 5. Effect of inlet distortion on the pressure ratio

In Fig. 6, the incidence angle related to the second stator at a mid-range mass flow rate is visible in $90 \%$ of speed lines, which shows its increase in case II. In case III, with the decreasing total pressure at the tip and increasing in the hub, totally different behavior is observed in Fig. 5. Surge and choking occur at lower mass flow rates and the surge margin is higher. One could say that the total pressure increase at the tip shifts pressure ratio curves to the right and its decrease shifts the curves to the left (compared to uniform inlet total pressure). The surge margin (as defined by Urasek et al. (1979)) is $11.7 \%$ in the design case (case I), $7.7 \%$ in case II with a greater total pressure at the tip and $12.9 \%$ in case III with a higher total pressure in the hub.

In the research conducted by Naseri and Boroomand (2016), the authors employed tip radial and hub radial patterns to model total-pressure distortion. They approached a tip radial distortion pattern by assuming its value in the blade hub area equal to the total pressure of clean inlet and its decreasing to a lower value at the blade tip area linearly. Alternatively, in the hub radial pattern, they set the total pressure in the blade tip area equal to the clean value and the 


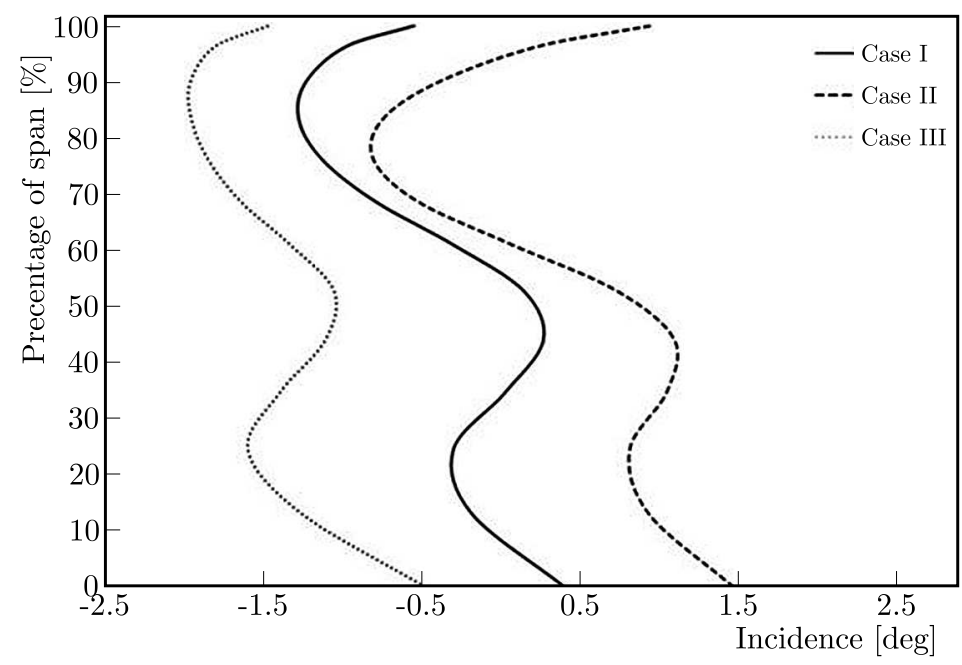

Fig. 6. Effect of inlet distortion on the incidence angle (mass flow $=29.9 \mathrm{~kg} / \mathrm{s}, N=0.9 N_{\text {des }}, 2$ nd stator)

low pressure side in the hub area. Their results indicated that hub radial distortion improves the operability range of the compressor. Therefore, the stall mass flow is reduced and the mass flow range between stall and choking conditions widened. The stall pressure ratio is also slightly reduced. Conversely, tip radial distortion reduces the operability range of the compressor. The mass flow range between choking and stall conditions is considerably reduced by increasing the intensity of tip radial distortion. Considering different definitions of radial distortion in Naseri's research, which leads to reduced averaged total pressure in different cases, their results confirmed the presented research findings. It should also be noted that with the decreasing rotational speed, lower absolute or relative Mach numbers are created. It can reduce the opportunity of surge and choking. Therefore, it is visible in Fig. 5 that the difference between the curves (three investigated cases) is higher at higher rotational speeds (except for $100 \%$ speed line in which SCM has higher computational errors). It is worth to mention that the prior research such as Liu et al. (2016), Naseri and Boroomand (2016), mainly focused on a specific rotational speed, and no study has been conducted for different revolution.

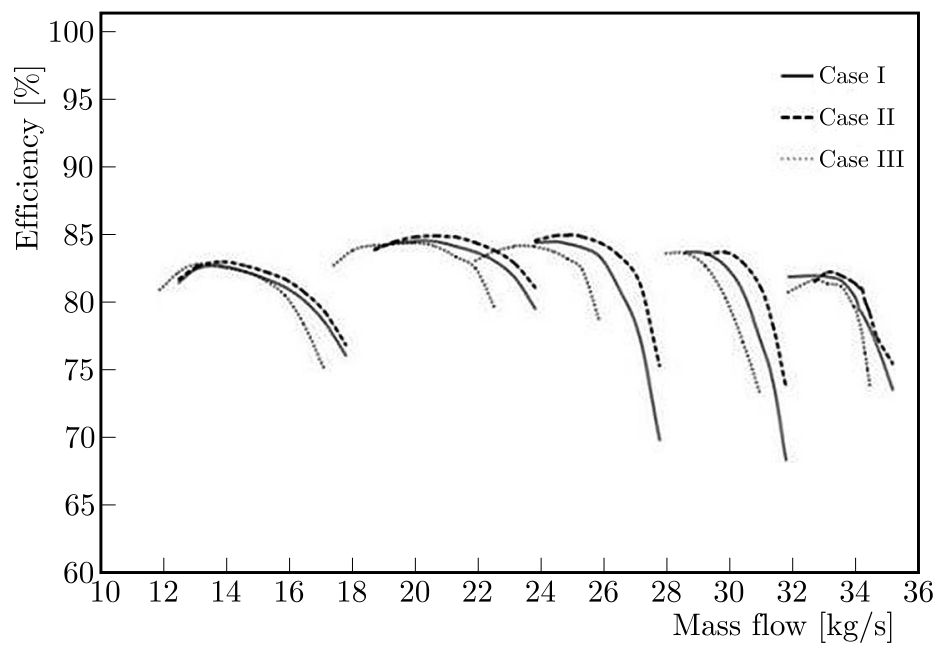

Fig. 7. Effect of inlet distortion on efficiency

Effects of inlet distortion on fan efficiency are also shown in Fig. 7 for the three cases. The previous statement about shifts in curves to the right or left due to distortions is also presented in Fig. 7. The results of the previous study (Naseri and Boroomand, 2016) just focused on the 
effects of distortion on the pressure ratio and its effect on the efficiency was not presented. The total pressure increase at the tip and a decrease in the hub (case II) produces higher efficiencies and the inverse case (case III) has opposite results. Also it could be seen that distortion effects are more significant in higher speed lines (except for 100\% speed line in which SCM has higher computational errors).

\subsection{Effects of inlet distortion on surge and choking}

In order to have a deeper understanding on the effects of inlet distortion on the compressor performance, some flow details are investigated. In this Section, the Mach number and the diffusion factor distribution for each case are presented in near surge condition. But, in similar studies, such results have not been extracted.

The diffusion factor can be considered as a criterion for the beginning of surge and stall. Therefore, in Fig. 8, a comparison between diffusion factors of different cases is carried out. The diffusion factor is plotted for the second stator at $90 \%$ speed line and the mass flow rate of surge beginning in the uniform flow case. A shift of the curves due to inlet distortions is also visible in Fig. 8. The total pressure increase at the tip and a decrease in the hub (case II), as stated in the previous Section, has weaker performance in the surge region. This behavior appears as the diffusion factor increases. This increase is higher in the hub and at the tip and is less in the mid-height, but there are higher diffusion factors in the whole blade height than in other cases. It can be seen that the case with a total pressure decrease in the hub and an increase at the tip (case III) has smallest diffusion factors and, therefore, better performance in the surge region.

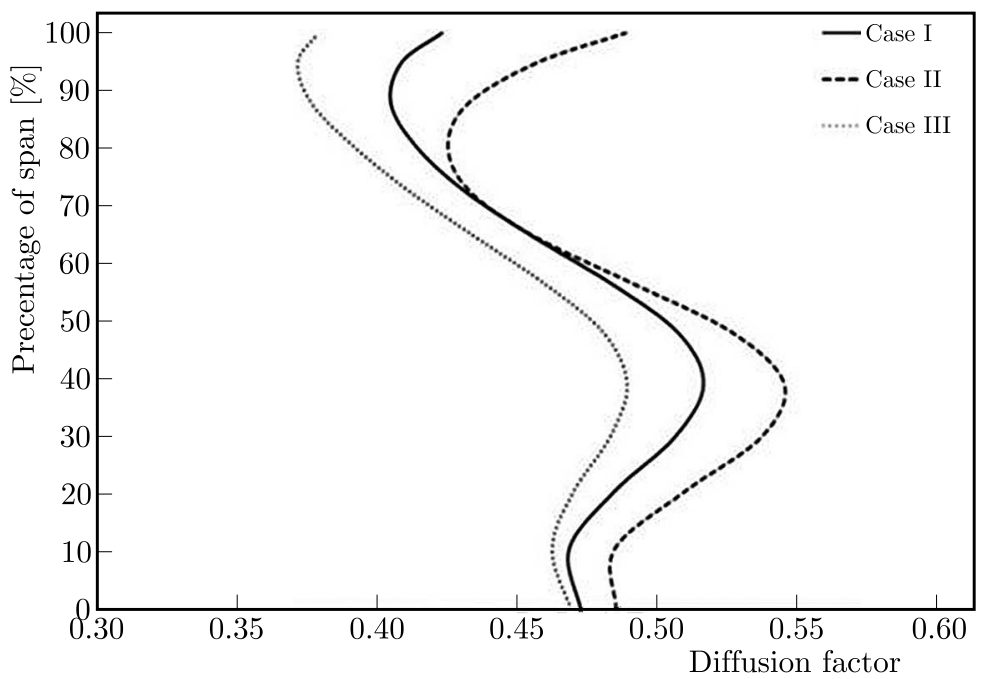

Fig. 8. Effect of inlet distortion on the diffusion factor (mass flow $=28 \mathrm{~kg} / \mathrm{s}, N=0.9 N_{\text {des }}$, 2nd stator)

Since, the Mach number is a criterion for choking prediction, a comparison between the Mach number for different investigated cases has been illustrated in Fig. 9. This figure compares the Mach number at the first stator inlet (with the highest inlet Mach numbers) at 90\% speed line and the mass flow rate at the beginning of choking in the uniform flow case. Referring to Fig. 9, the difference between the curves is small. It can be seen that the total pressure increase in the hub and a decrease at the tip (case III) creates higher Mach numbers in the total height of blade and, consequently, shows a weaker performance in the choking region. In this case, the Mach number in the hub of the first stator inlet is about 0.86 . So, it is a critical value that may increase to 1 in the blade throat and cause choking. 


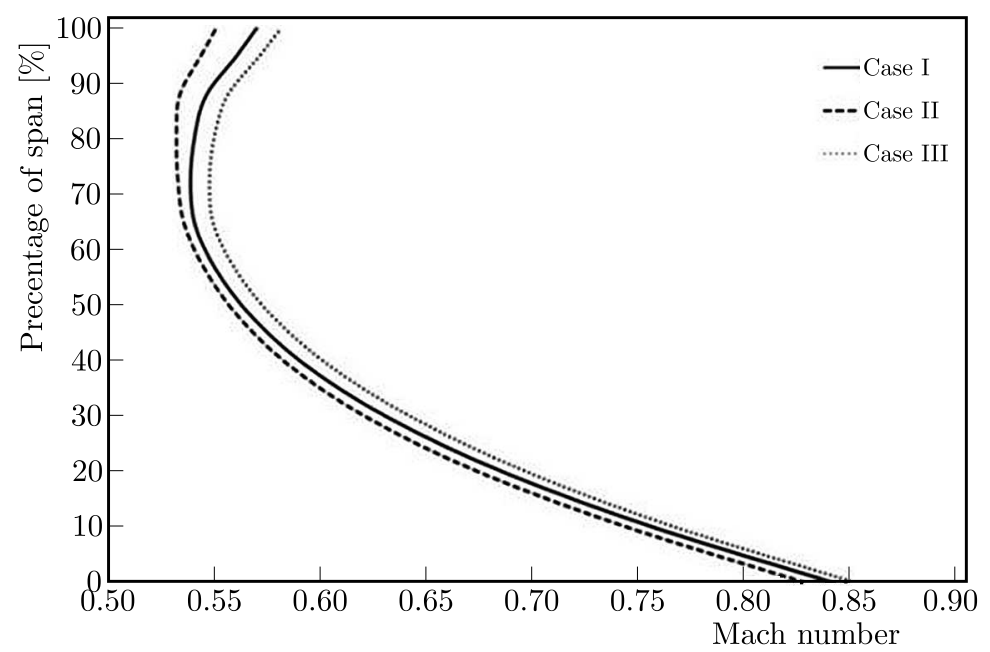

Fig. 9. Effect of inlet distortion on the Mach number (mass flow $=31.4 \mathrm{~kg} / \mathrm{s}, N=0.9 N_{d e s}$, 1 st stator)

\section{Conclusion}

Under a variety of conditions in military and commercial aircraft engines, inlet flow distortion produces many performance problems and engine failures including rotating stall and surge. Therefore, it is important to explore the behavior of the engine compressor towards inlet distortion. In this paper, the streamline curvature method (SCM) has been used to explore effects of inlet flow distortion on performance maps and change of surge/choke margins. This method compared with numerical (CFD) and experimental methods, can help avoiding costly and time consuming problems and indicates good accuracy and fast convergence. In order to achieve the purpose of the paper, radial variations of the total pressure set in a way that the average total pressure remains constant and equal to the design value. While, in a similar research, the average total pressure is not the same in different cases. The results show that the total pressure increase at the tip can shift performance curves to the right, increase the pressure ratio and efficiency, whereas its decrease shifts the curves to the left. The former case could lead to better performance in choking regions but reduces the surge margin. Conversely, the second case with the decreased total pressure at the tip and increased in the hub improved the surge margin. The research studies conducted to date are limited and mainly focus on the effect of distortion on the pressure ratio for a specific rotational speed and no study has been conducted in terms of both efficiency and different revolution speeds. Besides the aforementioned limitations, details of characteristics of the flow field such as flow angles, Mach number and the diffusion factor distribution have not been extracted from prior researches. But in the present research, these characteristics for each case are presented in a near surge condition under inlet total pressure distortion. The results indicated that variations of the second stator incidence and diffusion factor has only presented a weaker performance of the former case in surge regions. But variation of the first stator inlet Mach number has proved a weaker performance of the second case in choking regions.

\section{References}

1. Aungier R.H., 2003, Axial-Flow Compressors: a Strategy for Aerodynamic Design and Analysis, ASME Press, New York

2. Cetin M., Uecer A.S., Hirsch C., Serovy G.K., 1987, Application of Modified Loss and Deviation Correlations to Transonic Axial Compressors, Defense Technical Information Center, ADA191187 
3. Chue R., Hynes T.P., Greitzer E.M., Tan C.S., Longley J.P., 1989, Calculations of inlet distortion induced compressor flow field instability, International Journal of Heat and Fluid Flow, 10, 3, 211-223

4. Cumpsty, 1989, Compressor Aerodynamics, John Wiley \& Sons, New York

5. DAY I.J., 1993, Active suppression of rotating stall and surge in axial compressors, Journal of Turbomachinery, 115, 1, 40-47

6. Gong W.Q., Wu R.K., Zhang B., 2016, A new finite difference method to solve the velocity gradient equation in streamline curvature method, Advances in Mechanical Engineering, 8, 9, 1-13

7. Greitzer E.M., 1980, Review: axial compressor stall phenomena, Journal of Fluids Engineering, 102, 2, 134-151

8. Griepentrog H.R., 1970, Secondary flow losses in axial compressors, AGARD Lecture Series, 39

9. Hearsey R.M., 1986, Practical compressor aerodynamic design, Advanced Topics in Turbomachinery Technology, Principal Lecture Series, 2, Concepts ETI

10. Hu J.F., Ou-Yang H., Zhu X. Ch., Qiang X.Q., Du Z., 2011, An improved streamline curvature approach for transonic axial compressor performance prediction, Journal of Aerospace Engineering, 225, 5, 575-584

11. Kim J.H., Marble F.E., Kim C.J., 1996, Distorted inlet flow propagation in axial compressors, Proceedings of 6th International Symposium Transport Phenomena and Dynamics of Rotating Machinery, 2, 123-130

12. Kосн C.C., Smith L.H., 1976, Loss sources and magnitudes in axial flow compressors, Journal of Engineering for Gas Turbines and Power, 98, 3, 411-424

13. Lakshminarayana B., 1996, Fluid Dynamics and Heat Transfer of Turbomachinery, John Wiley \& Sons, New York

14. LiU H., WANG Y., XiAn S., Hu W., 2016, Effect of inlet distortion on the performance of axial transonic contra-rotating compressor, Journal of Aerospace Engineering, 10, 3, 1-13

15. Naseri M., Boroomand S., 2016, Numerical investigation of effect of inlet swirl and totalpressure distortion on performance and stability of an axial transonic compressor, Journal of Thermal Science, 25, 6, 501-510

16. NovaK R.A., 1967, Streamline curvature computing procedures for fluid-flow problems, Journal of Engineering for Power, 89, 4, 478-490

17. Pachidis V., Pilidis P., 2006, Prediction of engine performance under compressor inlet flow distortion using streamline curvature, Journal of Engineering for Gas Turbines and Power, 129, $1,97-103$

18. Storer J.A., Cumpsty N.A., 1994, An approximation analysis and prediction method for tip clearance loss in axial compressors, Journal of Turbomachinery, 116, 4, 648-656

19. SwAN W.C., 1958, A practical method of predicting transonic compressor performance, Journal of Engineering for Gas Turbines and Power, 83, 3, 322-330

20. Urasek D.C., Gorrell W.T., Cunnan W.S., 1979, Performance of a two stage fan having low aspect ratio first stage rotor blading, NASA Technical Paper, Report No. 1493

21. Zhang M., Hou A., 2017, Investigation on stall inception of axial compressor under inlet rotating distortion, Journal of Mechanical Engineering Science, 231, 10, 1859-1870 